\title{
Essential role of Ahnak in adipocyte differentiation leading to the transcriptional regulation of Bmpr1a expression
}

Jong Kyu Woo ${ }^{1}$, Jae Hoon Shin ${ }^{1,2}$, Seo Hyun Lee ${ }^{1}$, Hun-Min Park ${ }^{3}$, Soo Young Cho ${ }^{4}$, You Me Sung ${ }^{1}$, II Yong Kim and Je Kyung Seong ${ }^{1,3,5}$

\begin{abstract}
The role of Ahnak in obesity has been reported previously. Loss of Ahnak leads to decreased Bmp4/Smad1 signaling, resulting in the downregulation of adipocyte differentiation. However, the biological significance of Ahnak remains largely unknown. In this study, we demonstrate that Ahnak-mediated impaired adipogenesis results in decreased Bmprla transcriptional expression. To confirm this, Ahnak siRNA was used to knock-down Ahnak in C3H10T1/2 and primary stromal vascular fraction cells. Ahnak siRNA transfected cells showed suppression of Bmpr1a expression and decreased BMP4/ Bmpr1a signaling. The differential adipogenesis was further confirmed by knock-down of Bmpr1a in C3H10T1/2 cells, which resulted in reduced adipogenesis. Moreover, stable Ahnak knock-out C3H10T1/2 cells stably transfected with Ahnak CRISPR/Cas9 plasmid suppressed expression of Bmpr1a and prevented differentiation into adipocytes. Furthermore, we developed immortalized pre-adipocytes from wild-type or Ahnak Knock-out mice's stromal vascular fraction (SVF) to confirm the function of Ahnak in pre-adipocyte transition. Immortalized Ahnak knockout SVF cells showed lower level of Bmpr1a expression, evidence by their impaired BMP4/Bmpr1a signaling. Upon adipogenic induction, immortalized Ahnak knock-out SVF cells exhibited a marked decrease in adipocyte differentiation compared with immortalized wild-type pre-adipocytes. Furthermore, over-expression of Bmpr1a restored the adipogenic activity of Ahnak knock-out C3H10T1/2 cells and immortalized Ahnak knock-out SVF cells. Our data reveal the missing link in Ahnak-mediated adipose tissue remodeling and suggest that precise regulation of Ahnak in adipose tissue might have a therapeutic advantage for metabolic disease treatment.
\end{abstract}

\section{Introduction}

Obesity is characterized by the excessive adipocyte hypertrophy and hyperplasia caused by highly imbalance of energy expenditure vs. food intake ${ }^{1}$. Even though the clinical importance of obesity is growing, our understanding of the molecular mechanisms regulating the

Correspondence: Je Kyung Seong (snumouse@snu.ac.kr)

${ }^{1}$ Korea Mouse Phenotyping Center (KMPC), Seoul National University, Seoul, Republic of Korea

${ }^{2}$ Department of Surgery, University of Michigan, Ann Arbor, MI, USA

Full list of author information is available at the end of the article.

Edited by E. Candi adipocyte differentiation response to adipose tissue expansion is extremely limited. Thus, interest is focused on adipocyte differentiation as a platform for anti-obesity therapeutic approaches ${ }^{2}$.

Adipose tissues play a major role in energy storage in mammals. Adipose tissues have been divided into two distinct types: white adipose tissues (WATs), which are the primary site of energy storage by accumulating lipids and brown adipose tissues (BATs) ${ }^{3}$. Compared to WAT, the principal function of BAT is thermogenic energy consumption. WAT primarily contributes to energy storage for the regulation of energy balance and serves as a

\section{(c) The Author(s) 2018}

(c) Open Access This article is licensed under a Creative Commons Attribution 4.0 International License, which permits use, sharing, adaptation, distribution and reproduction cc) in any medium or format, as long as you give appropriate credit to the original author(s) and the source, provide a link to the Creative Commons license, and indicate if changes were made. The images or other third party material in this article are included in the article's Creative Commons license, unless indicated otherwise in a credit line to the material. If material is not included in the article's Creative Commons license and your intended use is not permitted by statutory regulation or exceeds the permitted use, you will need to obtain permission directly from the copyright holder. To view a copy of this license, visit http://creativecommons.org/licenses/by/4.0/. 
central endocrine organ playing key roles in metabolism ${ }^{4}$. Adipose tissue is comprised of adipocytes, which are the majority of cells in adipose tissue. Adipocytes are critical in normal physiology. The dysfunction of adipocytes causes a diverse range of diseases, including obesity, diabetes, and lipodystrophies ${ }^{5}$, derived by adipogenesis from specific precursor cells. The pre-adipocyte to adipogenic differentiation (adipogenesis) is a key process in fat mass. Adipogenesis requires an orchestrated multi-step process of sequentially concerted signaling pathways for adipocyte formation $^{6,7}$. The pre-adipocytes are derived from pluripotent mesenchymal stem cells (MSCs), which have the potential to commit to differentiate into adipocyte, myocyte, osteocyte, or chondrocyte lineages ${ }^{8}$, that become restricted to the adipocyte lineage through a multi-step procedure. Recruitment to this lineage gives rise to preadipocytes which undergo several stages and then differentiate into adipocytes 9 . The most critical transcription factors for adipogenesis are peroxisome proliferatoractivated receptor gamma (Ppar- $\gamma$ ) and CCAAT enhancer-binding protein alpha $(\mathrm{C} / \mathrm{EBP}-\alpha)$. Early signaling events mediated by $C / E B P-\beta$ and $C / E B P-\alpha$ contribute to initiate adipogenesis by inducing Ppar- $\gamma$, being both necessary and sufficient for white and brown adipocyte formation. In addition, they are also required to maintenance of the adipose tissues ${ }^{7,10-12}$.

Bone morphogenetic proteins (BMPs) were originally identified as factors that induce bone formation when implanted at ectopic sites ${ }^{13}$. Also, BMPs are transforming growth factor-beta (TGF- $\beta$ ) superfamily members that have pleiotropic functions during development by regulating embryogenesis, organogenesis, and morphogenesis $^{14,15}$. They signal through a hetero-tetrameric complex of transmembrane receptors known as type I and II serine/threonine kinase receptors ${ }^{16,17}$. Seven type I receptor members have been identified, which are referred to as activin-like kinase (ALK1-ALK7), while three members of type II receptors are known (BMPR2, ACTR2, and ACTR2B). Both type I and type II receptors contain an Nterminal extracellular-binding domain, a single transmembrane region, and an intracellular serine/threonine kinase domain ${ }^{16,18}$. Binding of BMP to the BMP receptor complex induces a signaling cascade. The two types of BMP-induced signaling pathways include the canonical Smad-dependent signaling pathway and Smad independent signaling pathway, such as p38 mitogen-activated protein kinase (MAPK) pathways ${ }^{18,19}$. Recently, it was reported that adipocyte differentiation is closely linked to Bmpr1 $\alpha /$ Smad1 signaling pathways ${ }^{20,21}$. BMP4/Bmpr1 $\alpha$ expression is a key regulator for the commitment and differentiation of pluripotent C3H10T1/2 cells to the white adipocyte lineage ${ }^{21,22}$. We recently reported that mice with genetic ablation of Ahnak exhibited reduced white adipocytes through impairment of BMP4-mediated
Smad signaling, which influences adipogenesis and glucose homeostasis ${ }^{23}$.

The neuroblast differentiation-associated protein, Ahnak, is an exceptionally large $(700 \mathrm{kDa})$ and ubiquitously expressed essential protein for cell proliferation and migration ${ }^{24-26}$. Ahnak is expressed in several intracellular locations, including the plasma membrane, cytoplasm, and nucleus ${ }^{27}$. Ahnak is highly expressed in adipose tissue and is upregulated in high-fat diet-induced obesity models ${ }^{28,29}$. Our previous studies have reported that Ahnak is involved in obesity and cellular adipogenesis process $^{23,30}$. However, the underlying molecular mechanism of Ahnak during adipogenesis is poorly understood.

We investigated whether Ahnak is involved in the programming of adipogenesis in pre-adipocytes. In the current study, we verified that Ahnak can control adipogenic differentiation by down-regulating Bmpr1 $\alpha$ expression in pre-adipocytes. The proposed inhibitory effect of Ahnak knock-out on white adipogenesis prompted us to further investigate the involvement of Bmpr1 $\alpha$ in adipocyte differentiation. Bmpr1 $\alpha$ overexpression restored Ahnak-mediated impairment of adipogenesis in pre-adipocytes. These data implicate Ahnak as a new regulatory factor that maintains proper adipogenic differentiation. Our findings reveal the mechanism by which Ahnak regulates adipocyte differentiation.

\section{Results}

Deficiency of ahnak downregulates bmpr1a expression in mouse adipose tissues

Previously, we reported the role of Ahnak in obesity and energy expenditure in mice model ${ }^{23}$. Deficiency of Ahnak leads to decreased amount of adipose tissues and resistance to obesity by the impairment of Smad signaling pathways. To address the molecular mechanism of Ahnak-mediated BMP4/Smad1 signaling impairment, we used microarray data blocked TGF-b/Smad3 signaling in WAT of Smad3deficient mice (GSE28598). Yadav et al. constructed a transcriptome profile for obesity and diabetes by blockade of TGF- $\beta /$ Smad3 signaling in WAT of Smad3-deficient mice $^{31}$. First, we selected 82 TGF- $\beta$ signaling pathwayrelated genes from the KEGG database. The co-expression patterns between Ahnak and the TGF- $\beta$ signaling pathway gene sets were identified by Pearson correlation ( $p$ value $<$ $0.05)$ and seven genes were selected. Several genes known to participate in the adipogenic process were correlated with Ahnak expression, including Bmpr1 $\alpha$, Id3, Ppp2ca, Smurf1, Thbs1, and Thbs2 (Fig. 1a). We found that the most correlatively expressed gene was Bmpr1 $\alpha$. We evaluated Bmpr1 $\alpha$ expression in iWAT. As shown in Fig. 1b, Bmpr1 $\alpha$ mRNA expression was reduced in iWAT from Ahnak knock-out (KO) mice compared to wild-type (WT) 
a

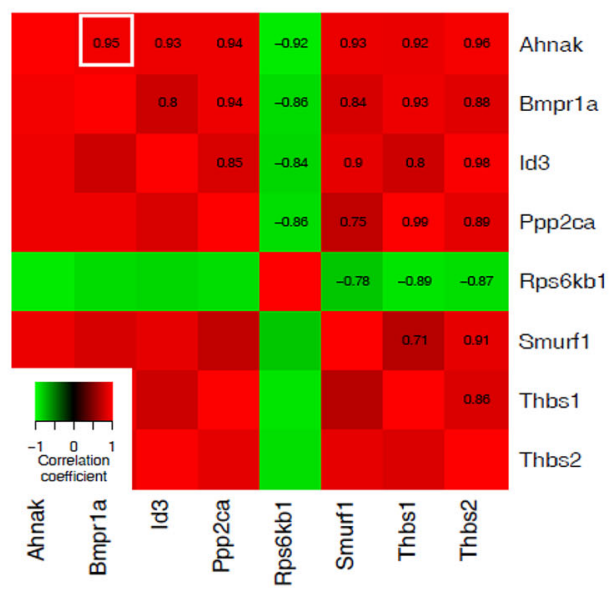

C H\&E
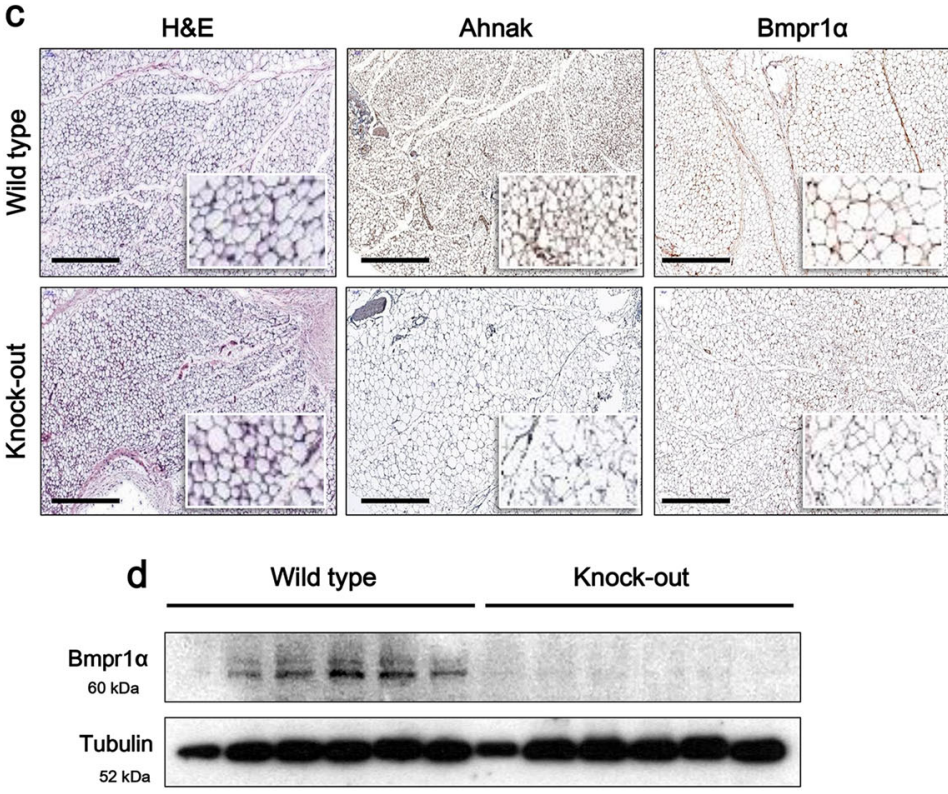
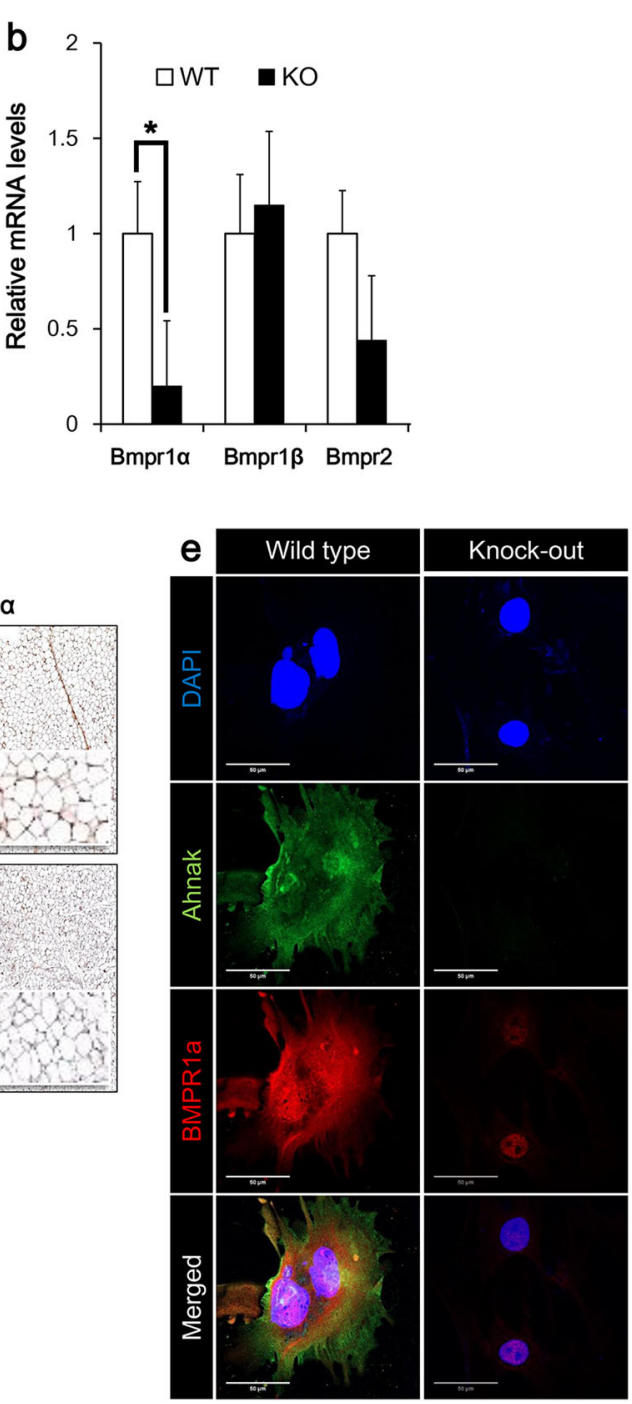

Fig. 1 Ahnak knock-out suppresses Bmpr1a expression. a The transcriptome profile from obesity and diabetes by blockade of TGF- $\beta$ / Smad3 signaling. The colors ranging from green to red represent Pearson correlation, indicating low to high correlations, respectively. $\mathbf{b}$ Relative expression of Bmpr1a was determined by qRT-PCR analysis (right panel). GAPDH serves as a loading control. Wild type (WT) and Ahnak knock-out (KO). c Immunohistochemical analysis of Ahnak or Bmpr1a in mouse inguinal white adipose tissues (iWAT). d Western blotting analysis of lysates harvested from wild-type and Ahnak Knock-out mice iWAT. e Immunofluorescence staining of Ahnak or Bmpr1a in primary SVF isolated from wildtype or Ahnak knock-out mouse iWAT

mice. In contrast, minor differences in Bmpr1 $\beta$ and Bmpr2 expression occurred in Ahnak $\mathrm{KO}$ mice. Furthermore, histological analysis revealed that Ahnak KO iWAT showed decreased expression of Bmpr1 $\alpha$ (Fig. 1c). Furthermore, Bmpr1 $\alpha$ protein levels are also measured by western blotting using mice iWAT (Fig. 1d). We confirmed that the Bmpr1 $\alpha$ expression was dependent on Ahnak expression in vivo. But, we could not detect Ahnak protein band since Ahnak protein size are larger than $700 \mathrm{kDa}$. Ahnak western blotting always showed poor band. Then, we performed indirect immunofluorescence (IF) analysis to examine the Ahnak and Bmpr1 $\alpha$ proteins expression in primary stromal vascular fraction (SVF), that were isolated from Ahnak knock-out or WT mice iWAT, and Ahnak and Bmpr1 $\alpha$ proteins were visualized by confocal microscope. Bmpr1 $\alpha$ expression was predominantly decreased in Ahnak knock-out SVF compared with WT SVF (Fig. 1e). Altogether, these results identified Ahnak as a marker expressed in SVF, with a positive correlation between Bmpr1 $\alpha$ mRNA and protein expression. 


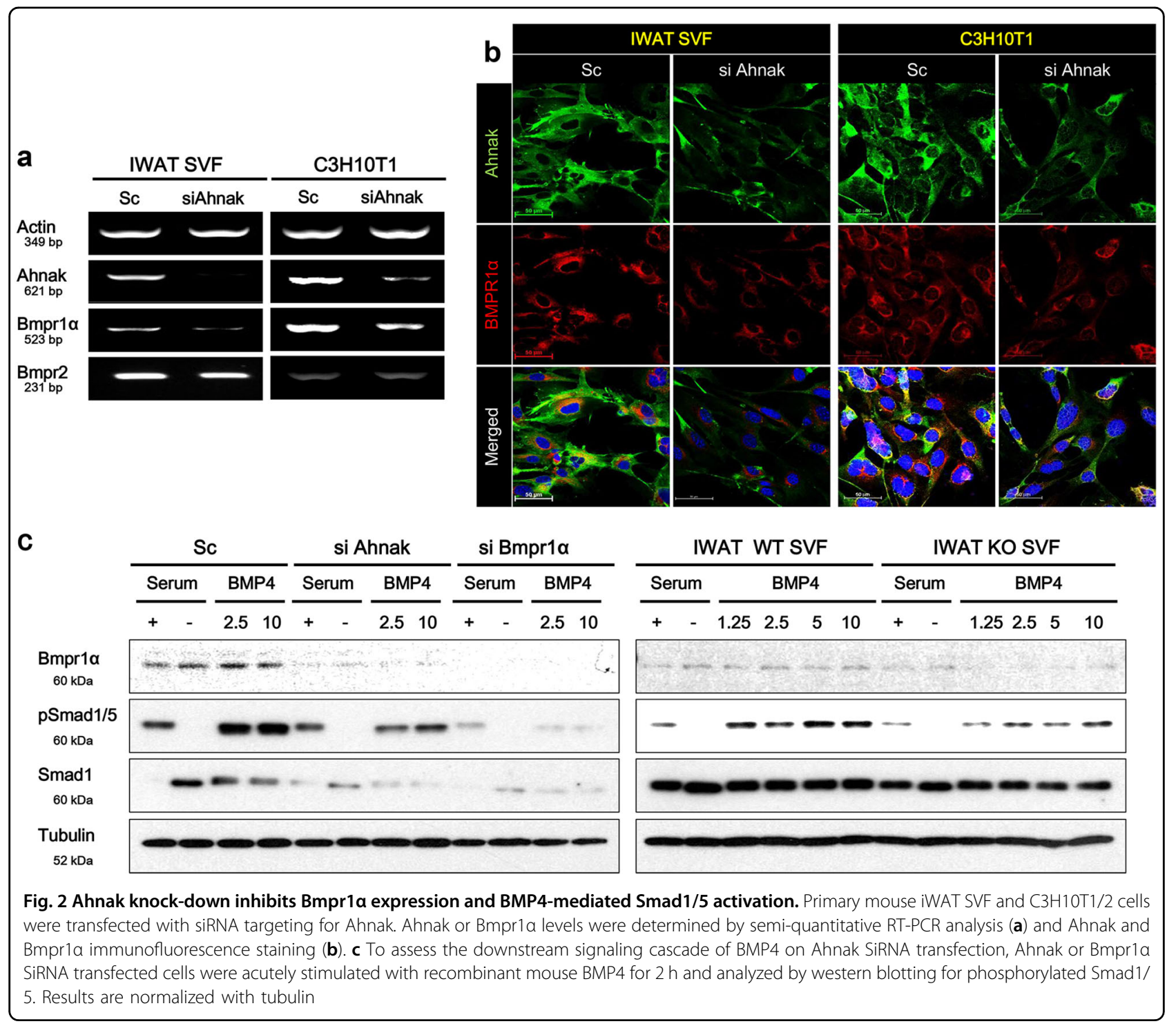

\section{Ahnak knock-down in C3H10T1/2 cell or primary SVF suppresses bmpr1a expression and BMP4/smad1/ 5 signaling pathway}

To confirm whether Ahnak expression directly modulates Bmpr1 $\alpha$ expression, we transfected Ahnak siRNA into $\mathrm{C} 3 \mathrm{H} 10 \mathrm{~T} 1 / 2$ cells or primary SVF cells, and tested the level of Bmpr1 $\alpha$ by RT-PCR and IF analysis. As Fig. 2a shows, Ahnak mRNA expression was downregulated in primary iWAT SVF and C3H10T1/2 cells by Ahnak siRNA. Ahnak depletion contributed to the downregulation of Bmpr1 $\alpha$ mRNA expression. However, Bmpr2 mRNA expression was not changed by Ahnak knock-down in two cell lines. It suggests that Ahnak may regulate Bmprl $\alpha$ specifically. The same results were observed in IF analysis. Primary iWAT SVF and C3H10T1/2 cells showed similarly reduced Bmpr1 $\alpha$ expression with Ahnak siRNA transfection (Fig. 2b).
Thus, we suggest that the Ahnak and Bmpr1 $\alpha$ expression might be linked in pre-adipocytes and participate in adipocyte maintenance or differentiation. Phosphorylation of Smad1/5 by the BMP4/Bmpr1 $\alpha$ signaling cascade regulates target gene transcription within the nucleus. Western blotting of whole cell lysate after $4 \mathrm{~h}$ of stimulation with BMP4 (2.5 or $10 \mathrm{ng} / \mathrm{mL})$ confirmed the lack of induction of Smad1/5 phosphorylation in Ahnak or Bmpr1 $\alpha$ deficiency cells. As shown in Fig. 2c (left panel), Ahnak siRNA or Bmprl $\alpha$ siRNA transfection resulted in an abrogation of BMP4-induced Smad1/5 phosphorylation in C3H10T1/2 cells. We next determined a Smad1/5 phosphorylation level in primary SVF between wild-type (WT) or Ahnak knock-out (KO) mice. SVF were collected from mice iWAT, cultured until reaching $80-90 \%$ confluence, and the cells were cultured in serum-free medium for $24 \mathrm{~h}$, then stimulated with sequential doses of BMP4 
for $4 \mathrm{~h}$ prior to western blotting analysis. Treatment of primary WT SVF with BMP4 resulted in phosphorylation of Smad1/5 but in Ahnak knock-out SVF, we observed reduced BMP4-stimulated Smad1/5 phosphorylation (Fig. 2c, right panel). This suggests the downregulation of Ahnak in pre-adipocytes suppresses Bmpr $1 \alpha$-mediating BMP4 signaling cascade that might have a pivotal role in the process of adipogenesis.

\section{Knock-down of ahnak or bmpr1a impairs adipogenic differentiation of $\mathrm{C} 3 \mathrm{H} 10 \mathrm{~T} 1 / 2$ cells}

Phosphorylated Smad1/5 are intermediates in the BMP4/Bmpr1 $\alpha$ signaling pathways of various cell types $^{18,19}$. To investigate the effect of Ahnak-mediated Bmpr1 $\alpha$ suppression on adipogenic differentiation of C3H10T1/2 cells, we knock-downed Ahnak or Bmpr1 $\alpha$ in C3H10T1/2 and compared their differentiation capacity with scrambled siRNA transfected cells. Cells at $80-90 \%$ confluence were transfected with Ahnak or Bmpr1 $\alpha$ siRNA and were treated to promote differentiation to white adipocytes. Ahnak or Bmpr1 $\alpha$ was required for lipid storage in differentiating C3H10T1/2 cells. Oil Red O staining revealed that knock-down of Ahnak or Bmpr1 $\alpha$ in the C3H10T1/2 cells displayed significant suppression of their differentiation capacity (Fig. 3a). Expression of adipogenic protein markers, such as peroxisome proliferator-activated Ppary and perilipin-1 (PLIN1), was significantly decreased in Ahnak or Bmpr1 $\alpha$ downregulated cells, as compared with scrambled controls (Fig. 3b, upper panels). Furthermore, mRNA levels also confirmed Ahnak-mediated or Bmpr $1 \alpha$-mediated impairment of adipogenesis (Fig. 3b, lower panels). Interestingly, we confirmed that the Bmpr1 $\alpha$ expression was dependent on Ahnak expression in C3H10T1/2 cells. The positive correlation between Ahnak and Bmprl $\alpha$ expression was seen only in Ahnak SiRNA transfection, but Bmpr1 $\alpha$ siRNA transfection did not alter Ahnak expression. Considering that Ahnak deficiency is likely to attenuate adipogenesis by suppression of Bmpr1 $\alpha$ expression, however, the behind mechanism of Ahnak deficiency mediates Bmpr1 $\alpha$ suppression in preadipocytes remains to be further investigated.

\section{Ahnak gene disruption with CRISPR/Cas9 on C3H10T1/2 cells suppressed BMP4/smad1/5 signaling pathways and white adipocyte differentiation}

To corroborate the effect that Ahnak exerts on white adipogenic differentiation, we used CRISPR/Cas9 gene disruption $^{32}$ to disrupt Ahnak using a single guide RNA approach in the C3H10T1/2 cells. From stable Ahnak CRISPR/Cas9 knock-out cell lines, termed Ahnak 1-9, we found that adipogenesis was significantly abolished (Fig. 4e) by Ahnak gene disruption, and was associated with loss of Bmpr1a protein (Fig. 4a). After selection of

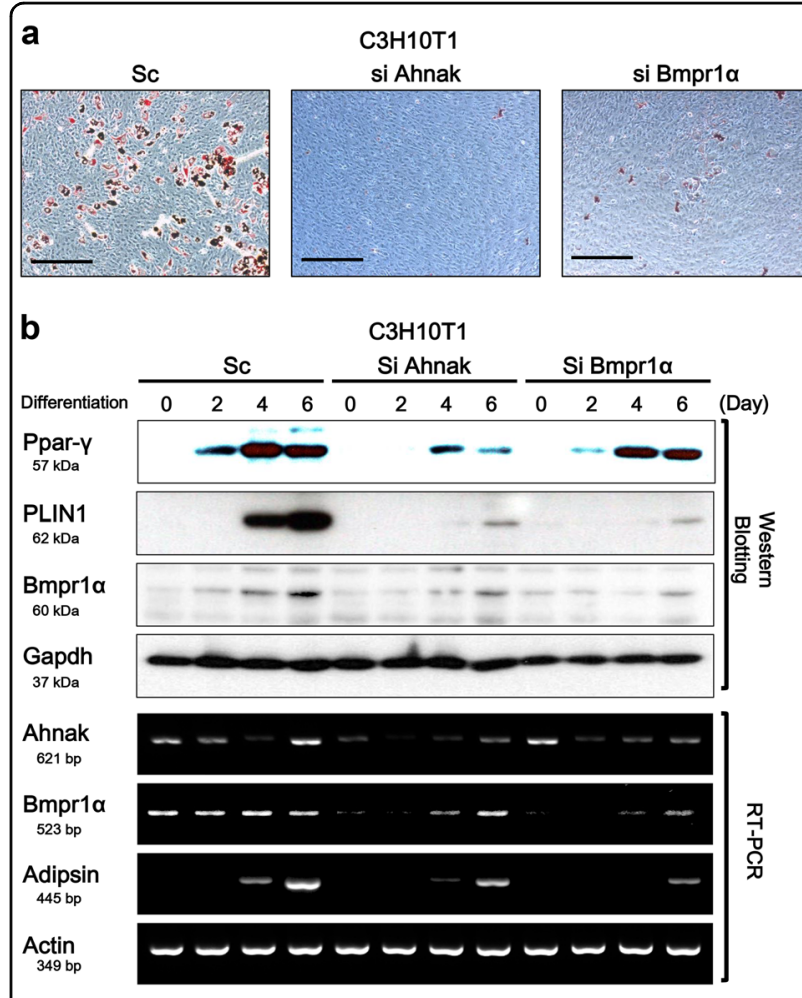

Fig. 3 Ahnak knock-down inhibits white adipocyte differentiation. $\mathrm{C} 3 \mathrm{H} 10 \mathrm{~T} 1 / 2$ cells were transfected with siRNA targeting Ahnak or Bmpr1a, and cells were assessed for standard white adipocyte differentiation. a Adipogenic differentiation was determined using Oil Red O staining. b Protein or mRNA level of white adipogenic markers were analyzed by western blotting and semiquantitative RT-PCR

Ahnak knock-out cells, Ahnak 1-9, demonstrated 45\% reduction of Bmpr1 $\alpha$ proteins expression as shown by FACS analysis. In addition, immunofluorescence analysis showed Bmpr1 $\alpha$ downregulation in Ahnak 1-9 cells (Fig. 4b). Given the critical roles of Bmpr1 $\alpha$ in adipogenesis and Smad1/5 signaling cascades, we examined whether Smad1/5 phosphorylation was inhibited by Ahnak gene deletion. As shown in Fig. 4c, Smad1/5 was activated by presence of BMP4 in C3H10T1/2 control cells, but BMP4-mediated Smad1/5 phosphorylation was blocked by Ahnak disruption in Ahnak 1-9 cells. Ahnak gene disruption also inhibited induction of Ppar- $\gamma$ and $\mathrm{C} /$ EBP $\alpha$ in white adipogenesis stimulation (Fig. 4d). In addition, Ahnak 1-9 cells displayed decreased fat accumulation when white adipocyte differentiation was induced compared with the C3H10T1/2 control cells as demonstrated by reduced PLIN 1 immunofluorescence staining (Fig. 4e). Altogether, these results indicated that Ahnak gene disruption subsequently downregulated the expression of Bmpr1 $\alpha$, together with BMP4-mediated Smad1/5 activation, thus inhibiting the adipogenic differentiation of $\mathrm{C} 3 \mathrm{H} 10 \mathrm{~T} 1 / 2$ cells. 


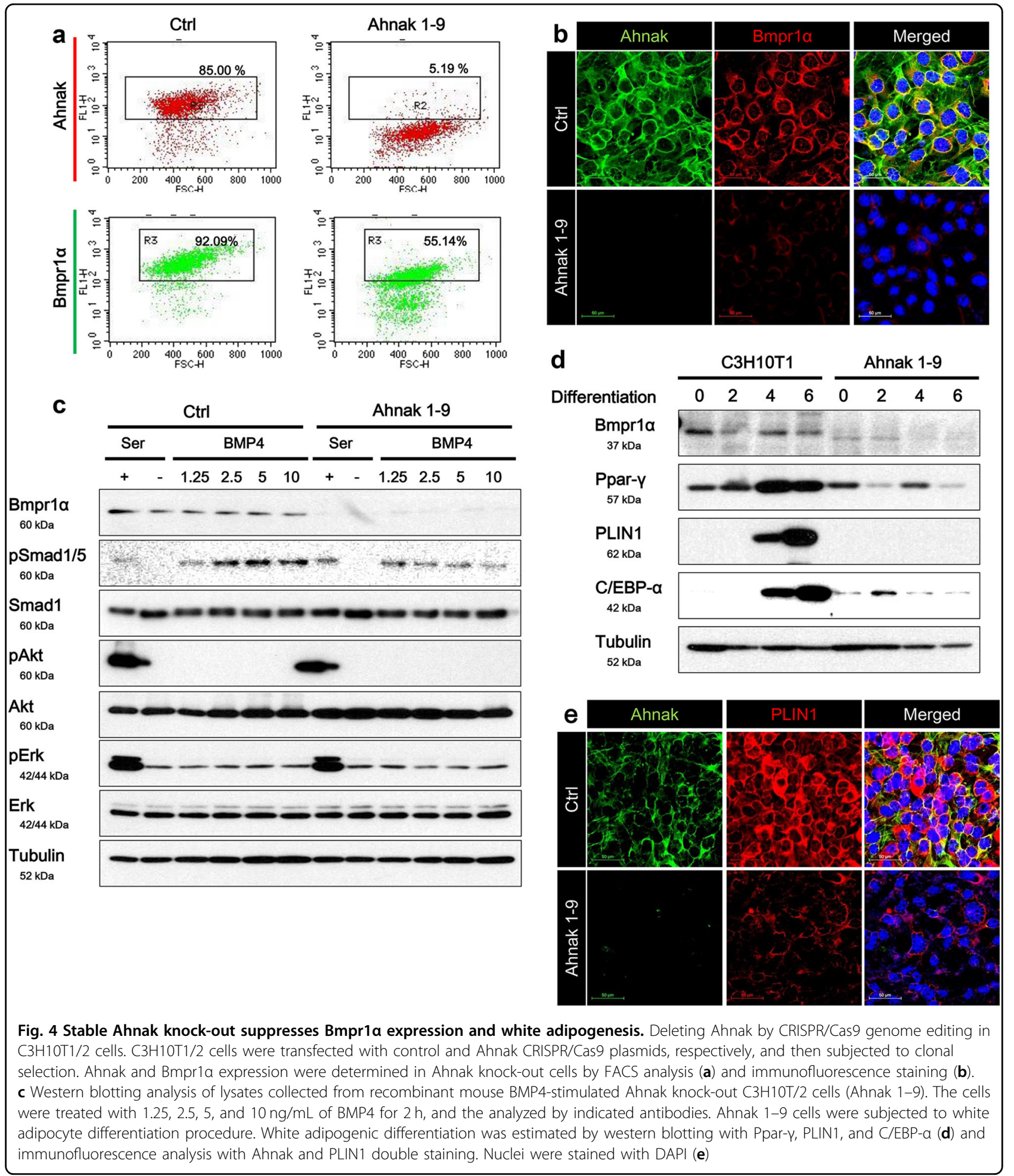

Immortalized iWAT SVF cells showed different adipogenic differentiation capacity

To obtain unbiased insight into putative Ahnakdependent molecular pathways impacting Bmpr1 $\alpha$ mediated adipocyte differentiation, we developed immortalized pre-adipocytes derived from SVF of WT, Ahnak hetero zygote ( $\mathrm{He})$, and Ahnak knock-out mice iWAT (Im WT, Im He, and Im Ko, respectively). The immortalized cells were passaged in culture for more than 30 days and were followed for up to 10 passages. The 
induction of adipocyte differentiation was tested through the use of a standard protocol for white adipogenesis that employs a cocktail of insulin, dexamethasone, and 3isobutyl-1-methylxanthine (IBMX). Having established that immortalized cells were capable of differentiating into adipocytes in vitro and that the immortalized cells retained differentiation characteristics of primary cells, we performed fluorescent-activated cell sorting (FACS) analysis to verify Ahnak and Bmpr1 $\alpha$ expression in immortalized cells. FACS analysis revealed that $10.7 \%$ of cells expressed Ahnak and Bmpr1 $\alpha$ together in Im WT cells. The Ahnak/Bmpr1 $\alpha$ double-positive population comprised approximately half of the cells population (5.42\%) present in the Im He cells compared as Im WT cells, but Im Ko cell was negative for Ahnak and Bmpr1 $\alpha$ doublepositive population. Indeed, Ahnak negative Bmpr1 $\alpha$ positive populations were similar among all three cell lines (Fig. 5a). These results unambiguously demonstrate the role of Ahnak protein, which controls Bmprl $\alpha$ expression in SVF cells. Bmpr1 $\alpha$ mRNA expression was further confirmed by RT-PCR. Bmpr1 $\alpha$ downregulation in Im $\mathrm{He}$ and Im Ko cells provided evidence of the regulatory role of Ahnak (Fig. 5b). Additionally, immortalized SVFs were immunofluorescently stained by a specific antibody for Ahnak and Bmpr1 $\alpha$ and were analyzed using confocal laser microscopy (Fig. 5c). As expected, Im WT cells retained an identical staining pattern of Ahnak and Bmpr1 $\alpha$. In contrast, the Im Ko cells showed a poor staining pattern of Ahnak and Bmpr1 $\alpha$ under basal conditions. Consistent with FACS and mRNA expression data, Bmpr1 $\alpha$ protein was decreased in Im Ko cells by IF analysis. Western blotting with the antibodies that recognizes the Bmpr1 $\alpha$ and phosphorylated Smad1/5 revealed a robust decrease in BMP4-stimulated phosphorylation in Im Ko cells compared as Im WT cells. Nonetheless, BMP4-stimulated phosphorylation of Smad1/5 was almost abolished in Im Ko cells with no change in total Smad1 protein or the Actin protein (Fig. 5d). To confirm the role of Bmpr1 $\alpha$ on BMP4stimulated phosphorylation of Smad1/5, Im WT cells were transfected with Bmpr1 $\alpha$ SiRNA and then stimulated with BMP4. BMP4-stimulated phosphorylation of Smad1/5 was also significantly decreased with no change in Smad1 protein (Fig. 5d). SVF cells of adipose tissue are characterized by their capacity to differentiate into mature adipocytes $^{33}$. The immortalized SVF cells were subjected to adipogenesis. When the accumulation of intracellular lipids was confirmed by Oil Red O staining, the staining intensity was decreased in Im Ko cells. In addition, PLIN1 staining was also decreased in Im Ko cells during adipogenesis (Fig. 5e). Expression of adipogenic markers, such as Ppar- $\gamma$ and C/EBP $\alpha$ proteins and mRNA, were significantly decreased in Im Ko cells compared with Im WT (Fig. 5f). These results revealed an apparent decrease in the expression of Bmpr1 $\alpha$ in Ahnak 1-9 and Im Ko cells (Figs. 4 and 5). These results indicate that Ahnak deficiency-mediated suppression of Bmpr1 $\alpha$ occurred at the transcriptional level through the downregulation of transcriptional activity. As showed in Fig. 6a, b, Ahnak deficiency directly targeted Bmpr1 $\alpha$ promotor transcriptional activity. Taken together, the results unambiguously demonstrate the necessity of Ahnak in white adipogenesis whether upregulated Bmpr1 $\alpha$ expression for preadipocytes differentiation by BMP $4 / \mathrm{Smad} 1 / 5$ signaling cascade.

\section{Over expression of bmpr1a restores ahnak deficiency- mediated adipogenesis impairment}

To assess functional involvement of Bmpr1 $\alpha$ in Ahnak deficiency-mediated adipogenesis impairment, we applied ectopic mouse Bmpr1 $\alpha$ expression in Ahnak knock-out C3H10T1/2 cells (Ahnak 1-9) and Immortalized Ahnak knock-out SVF cells (Im Ko). Western blotting analysis demonstrated a clear increase of Bmpr1 $\alpha$ expression in the Bmpr1 $\alpha$ introduced Ahnak 1-9 cells. Then, we determined the functional effects of ectopic expressed Bmpr1 $\alpha$ by analyzing the downstream signaling upon BMP 4 stimulation. The Bmpr1 $\alpha$ introduced Ahnak 1-9 cells showed increased phosphorylation of the Smad1/5 by BMP 4 stimulation (Fig. 7a). Furthermore, IF staining of these Bmpr1 $\alpha$ transfected Ahnak 1-9 cells showed an intense Bmpr1 $\alpha$ staining in contrast to the control vector transfected Ahnak 1-9 cells (Fig. 7c, left panel). Thus, ectopic Bmpr1 $\alpha$ over-expression repaired the impaired adipocyte differentiation caused by Ahnak deficiency as assessed by the expression of the adipogenesis specific marker proteins Ppar- $\gamma$ and PLIN1 (Fig. 7b). These findings were confirmed by IF analysis (Fig. 7c, right panels); PNL1 expression was much higher in Bmpr1 $\alpha$ transfected Ahnak 1-9 cells. In addition, we examined whether Bmpr1 $\alpha$ also contributes to adipogenic impairment in Im Ko cells. The cells were transiently transfected with eGFPconjugated mouse Bmpr1 $\alpha$. As expected, the levels of Ppar- $\gamma$, PLIN1, and C/EBP1 $\alpha$ were increased in Im Ko cells (Fig. 7d, e). Thus, Bmpr1 $\alpha$ expression overcomes Ahnak deficiency related adipogenesis impairment in preadipocytes. The results corroborated the functional role of Ahnak during adipogenic differentiation mediated through the Bmpr1 $\alpha$ signaling pathway.

\section{Discussion}

Ahnak plays a pivotal role in adipocyte differentiation. We previously found that Ahnak functions in obesity resistance and insulin sensitivity by regulation of Smad1/ 5 signaling pathways. Genetic depletion of Ahnak protects from obesity and enhances insulin sensitivity ${ }^{23}$. Here, we show that Ahnak deficiency in pre-adipocytes negatively regulates Bmpr1 $\alpha$ expression and adipogenic 


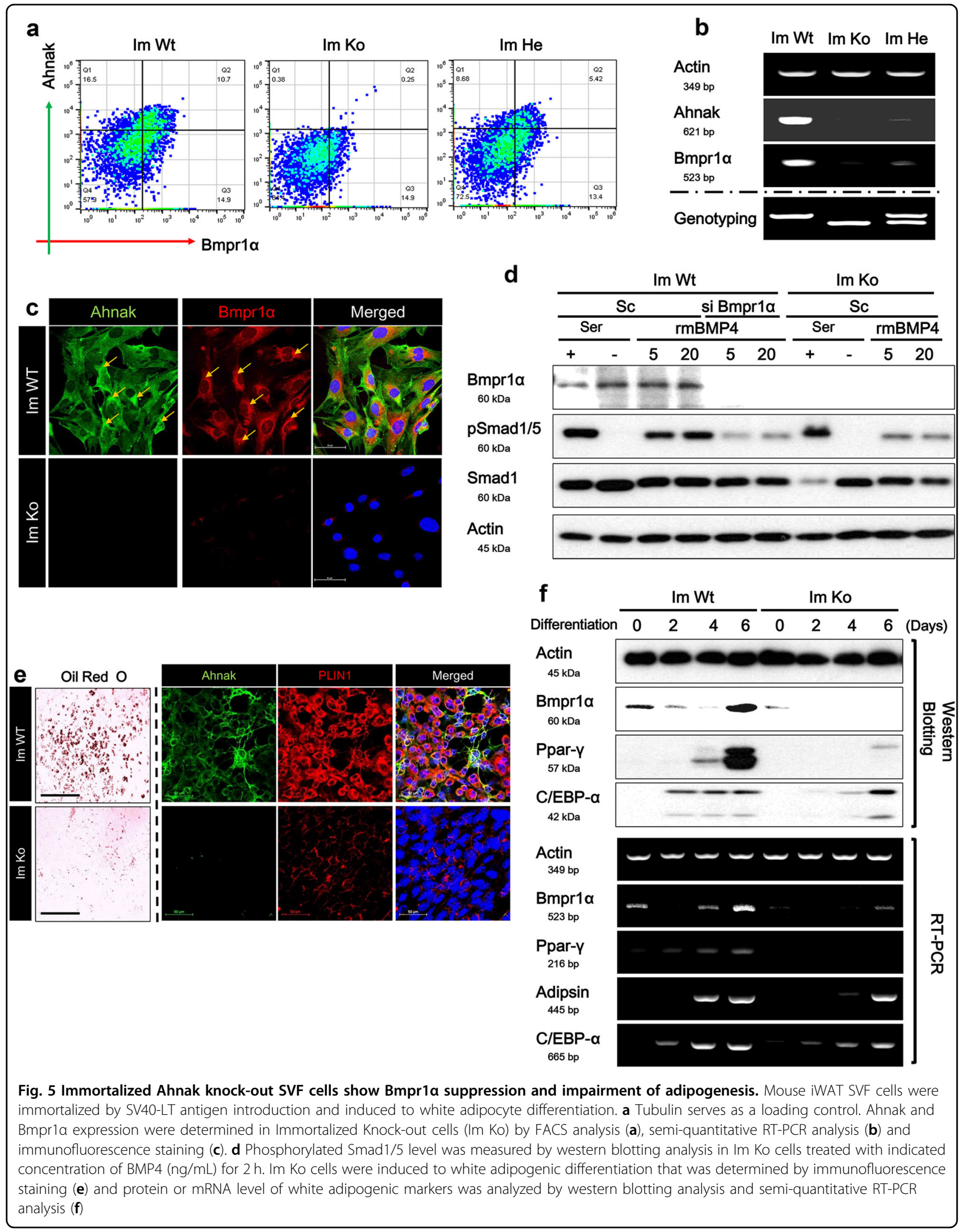


Immortalized SVF

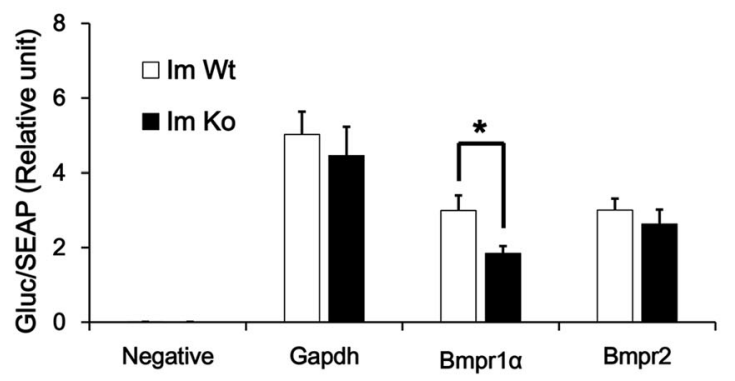

C3H10T1

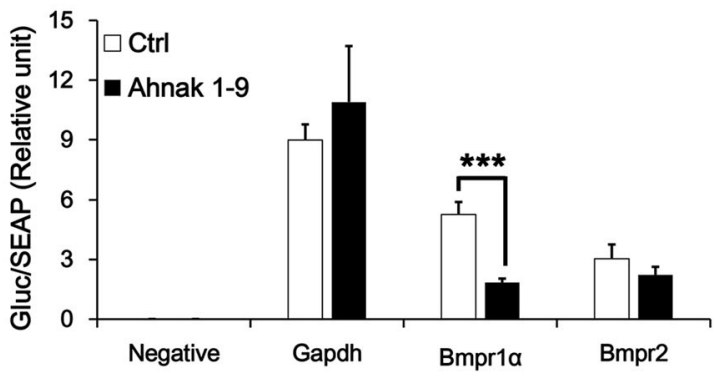

Fig. 6 Ahnak regulates Bmpr1a transcriptional activity. The immortalized Ahnak SVF (left panel) and Stable Ahnak knock-out (right panel) and cells were transfected with indicated plasmid encoding mouse GAPDH promoter region $(-1333 /+13)$, Bmpr 1a promoter region $(-1012 /+366)$, and Bmpr2 promoter region $(-1492 /+179)$ with gaussia luciferase construct. Cells were transfected for 2 days and supernatant were collected for the luciferase assays. Luciferase activity was normalized to secreted alkaline phosphatase. Data are presented as the mean \pm s.d. ${ }^{* *}$ or ${ }^{* * *}$ indicates a $p$ value 0.01 or 0.001 by Student's t-test analysis

differentiation. Adipogenesis is a step-wise process. Preadipocyte first undergo growth arrest, re-enter the cell cycle under the influence of differentiation inducers, undergo mitotic clonal expansion and, finally, exit the cell cycle to undergo terminal differentiation ${ }^{9,34}$. BMP/Smad signaling pathways play pivotal roles in terminal differentiation from the pre-adipocytes into adipocytes ${ }^{20,22}$. In this research, we discovered that Bmpr1 $\alpha$, the adipogenesis regulator, was significantly linked with Ahnak in mouse adipose tissues. Emerging evidence showed that Ahnak knock-out inhibits the transcriptional activity of Bmpr1 $\alpha$ in adipose tissues. As shown in Fig. 1b, the mRNA level of Bmpr1 $\alpha$ was decreased in iWAT of Ahnak knock-out mouse. We also showed decreased Bmpr1 $\alpha$ expression in SVF (Fig. 1d). Then, we showed that transient Ahnak knock-down suppresses Bmpr1 $\alpha$ expression and BMP4-dependent Smad1/5 phosphorylation (Fig. 2). A previous study demonstrated that loss of Ahnak suppresses Smad1/5 phosphorylation. Our results support the view that Ahnak-mediated Bmpr1 $\alpha$ suppression regulates adipogenesis by modulating BMP4-dependent Smad1/5 signaling. Our data demonstrate that Ahnak is required for maintaining of Bmpr1 $\alpha$ expression for preadipocyte differentiation.

Ahnak knock-down inhibited differentiation of C3H10T1/2 cells to white adipocytes as measured by Ppar- $\gamma$ and PLIN1 levels. In support of our findings, knock-down of Bmpr1 $\alpha$ also impaired white adipogenesis. Of note, Ahnak deficiency-mediated adipogenesis inhibition was independent from altered cell viability (data not shown). Thus, Ahnak protein acts as a regulator that promotes the differentiation of pre-adipocytes to white adipocytes via Bmpr1 $\alpha$ expression. Although it has been reported that Ahnak knock-out mice display obesity resistance and elevated energy expenditure, the under- lying molecular mechanism is unclear ${ }^{23}$. In this study, we reveal the molecular links between Ahnak and Bmprl $\alpha$ in relation to white adipogenesis.

Establishment of stable transfected C3H10T1/2 cells with Ahnak CRIPSR/Cas9 plasmid prevented differentiation into white adipocytes. The cells remained spindle shaped, while control cells became white adipocytes, indicated by Ppar- $\gamma$, C/EBP- $\alpha$, and PLIN1 measurements. Furthermore, we developed immortalized Ahnak knockout SVF cells and subjected them to standard white adipogenic differentiation process in vitro. Ahnak knock-out SVF cells showed consistent and significantly decreased BMP4/Smad1/5 signaling pathways and white adipogenic differentiation. The differential white adipogenesis was further confirmed by IF staining and decreased Ppar- $\gamma, \mathrm{C} /$ EBP- $\alpha$, and PLIN1 expression. These data demonstrate that Ahnak negatively regulates white adipogenic differentiation of pre-adipocytes.

Thereafter, we investigated whether over-expression of Bmpr1 $\alpha$ recovered Ahnak deficiency effect in Ahnak knock-out pre-adipocytes. We examined the stable Ahnak knock-out C3H10T1/2 cells (Ahnak 1-9) or immortalized Ahnak knock-out SVF cells (Im Ko). Mouse Bmpr1 $\alpha$ over-expressing cells showed enhancement of the white adipogenesis compared with mock control cells. White adipogenic marker proteins Ppar- $\gamma, \mathrm{C} / \mathrm{EBP}-\alpha$, and PLIN1 were significantly increased, and BMP4-dependent Smad1/5 activation was enhanced (Fig. 7). In addition, accumulation of intracellular lipid droplets were increased in Bmprl $\alpha$ over-expressing Ahnak knock-out cells, as shown by PLIN1 IF staining.

Ahnak deficiency suppresses the expression of Bmpr1 $\alpha$ and the BMP4/Smad1/5 signaling pathway to initiate adipogenic differentiation. Our findings demonstrate that: (1) Ahnak knock-out mice adipose tissues display 


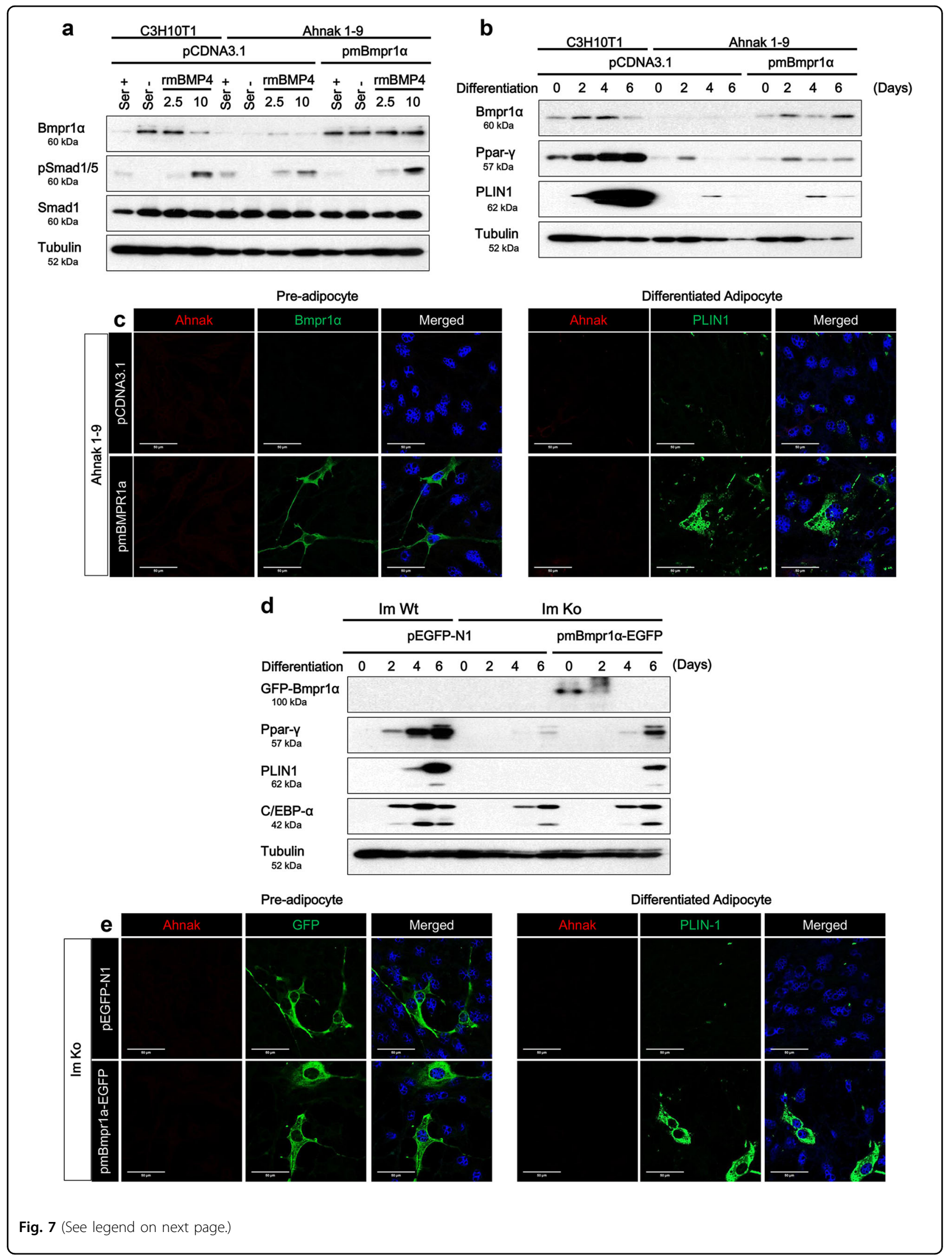


(see figure on previous page)

Fig. 7 Bmpr1a over-expression induces white adipogenesis in Ahnak knock cells. Ahnak knock-out C3H10T1/2 (Ahnak 1-9) cells were transfected with mouse Bmpr1a encoded plasmid. Transfected cells were stimulated with indicated concentration of recombinant mouse BMP4 (ng/ $\mathrm{mL}$ ) for $2 \mathrm{~h}$. (a) Western blotting analysis was performed with indicated antibodies. Tubulin was used as an internal control. After transfection, white adipocyte differentiation was induced. The effect of Bmprla on adipogenesis in Ahnak deficiency cells were assessed by western blotting analysis (b) and immunofluorescence analysis with Ahnak and PLIN1 double staining. Nuclei were stained with DAPI (c). Immortalized Ahnak knock-out (Im Ko) cells were subjected upon Bmpr1a over-expression. The effect of Bmpr1a conjugated eGFP on adipogenesis were determined by western blotting analysis (d) and immunofluorescence analysis with Ahnak and PLIN1 double staining. Nuclei were stained with DAPI (e)

decreased Bmpr1 $\alpha$ transcriptional activity; (2) knock-out of Ahnak inhibits Bmpr1 $\alpha$ expression and adipogenesis in C3H10T1/2 cells; (3) blockage of Smad1/5 signaling pathways by knock-out of Ahnak in C3H10T1/2 or SVF induced by BMP4; (4) knock-out of Ahnak prevents adipogenic differentiation in $\mathrm{C} 3 \mathrm{H} 10 \mathrm{~T} 1 / 2$ or SVF cells; and (5) gain-of-function experiments showed that Bmprl $\alpha$ expression recovered white adipogenesis in Ahnak knockout pre-adipocyte cells. Upon BMP4 stimulation, Bmpr1 $\alpha$ becomes activated and phosphorylates regulatory Smad1/ 5. The activated Smad1/5 is then translocated to the nucleus and binds to target gene promoters to regulate transcription of various adipogenesis regulating genes.

As Ahnak deficiency mediates obesity resistance, we proposed possible mechanism that Ahnak cooperates with Bmpr1 $\alpha$, which regulates the BMP $4 / \mathrm{Smad} 1 / 5$ signal transduction. Taken together, our data provide evidence that Ahnak proteins in the adipose tissues control adipocyte differentiation functions through Bmpr1 $\alpha$ mediated BMP $4 / S m a d 1 / 5$ signaling pathways. These findings may have therapeutic implications for the treatment of obesity or related metabolic diseases, such as cachexia and lipodystrophy.

\section{Materials and methods Antibodies and materials}

Antibodies to $\beta$-actin, tubulin, glyceraldehyde-3phosphate dehydrogenase (GAPDH), and enhanced green fluorescent protein (eGFP) and Ahnak CRISPR/ Cas9 plasmid were purchased from Santa Cruz Biotechnology (Santa Cruz, CA, USA). Anti-Ahnak and Bmpr1 $\alpha$ antibodies were obtained from Abcam (Cambridge, UK). Anti-Ppar- $\gamma$, perilipin-1 (PLIN1), phosphorylated Smad1/5 (pSmad1/5), Smad1, phosphorylated AKT (pAKT), AKT, phosphorylated extracellular signalregulated kinase (ERK)1/2 (pERK1/2), ERK1/2 and C/ $E B P \alpha$ antibodies were purchased from Cell Signaling Technology (Beverly, MA, USA). 3-Isobutyl-1methylxanthine (IBMX) and triiodothyronine (T3) were obtained from Cayman Chemical (Ann Arbor, MI, USA), Dexamethasone (DEX), rosiglitazone (Rosi), insulin, and 0.5\% Oil Red O were purchased from Sigma-Aldrich (St. Louis, MO, USA). Fetal bovine serum, Dulbecco's phosphate buffered saline (DPBS), Dulbecco's modified Eagle's medium (DMEM), and DMEM/F12 were purchased from WelGene (Daegu, Korea). Secrete-Pair Dual Luminescence Assay Kit and pEZX-Bmpr1 $\alpha$ promoter plasmid were obtained from GeneCopoeia (Rockville, MD, USA)

\section{Stromal vascular fraction cells isolation and immortalization}

SVF was isolated from inguinal white adipose tissue (iWAT) of 6-week-old male Wild-type or Ahnak Knockout mice ${ }^{35}$. Briefly, the adipose tissue was digested with 0.075\% collagenase type II (Worthington Biochemical Corporation, Lakewood, NJ, USA) containing $1 \%$ bovine serum albumin (BSA) for $2 \mathrm{~h}$ at $37^{\circ} \mathrm{C}$ with shaking. Following complete digestion, the mature adipocytes were separated from the SVF by centrifugation at $1200 \mathrm{~g}$ for 10 $\mathrm{min}$. The floating mature adipocytes were discarded, and the precipitated SVF cells were re-suspended with $1 \times$ red blood cell (RBC) lysis buffer (eBioscience, ThermoFisher, Waltham, MA, USA) for $10 \mathrm{~min}$ at room temperature. After RBC lysis, SVF were passed through $40 \mu \mathrm{m}$ cell strainers (SPL Life Sciences, Pocheon, Gyeonggi, Korea) and the cells were washed twice with DPBS. Isolated SVF cells were cultured in DMEM/F12 containing 10\% FBS and antibiotics. For immortalization, cells at $40-60 \%$ confluency were transfected with SV40 LT/pSG5 viral construction, and $5 \mu \mathrm{g} / \mathrm{ml}$ puromycin was added to select the stable cells. All mice were maintained under specific pathogen-free conditions in the institutional animal facility of the College of Veterinary Medicine, Seoul National University. The experiments were performed according to the "Guide for Animal Experiments" (Edited by Korean Academy of Medical Sciences) and approved by the Institutional Animal Care and Use Committee (IACUC) of the Seoul National University (Permit Number: SNU-130903-1).

\section{In vitro adipogenesis and oil red $\mathrm{O}$ staining}

C3H10T1/2 cells and immortalized pre-adipocytes were grown to confluency for 2 days and were cultured in white induction medium containing the $5 \mu \mathrm{g} / \mathrm{mL}$ insulin, $1 \mu \mathrm{M}$ DEX, $0.5 \mu \mathrm{M}$ IBMX, and with or without $0.5 \mu \mathrm{M}$ Rosi. After 2 days, cells were maintained in DMEM/ F12 supplemented with $10 \% \mathrm{FBS}, 5 \mu \mathrm{g} / \mathrm{mL}$ insulin, and 1 $\mathrm{nM}$ T3 until the end of the experiments. After 
differentiation, the adipocytes were visualized by Oil Red $\mathrm{O}$ staining. Cells were washed three times with PBS and fixed for $15 \mathrm{~min}$ with $4 \%$ paraformaldehyde. Oil Red O solution $(0.5 \%)$ freshly diluted into distilled water at a ratio of 3:2 was incubated with the fixed cells for $1 \mathrm{~h}$ at room temperature. Cells were washed with water and the stained lipid droplets in the adipocytes were visualized by light microscopy and photographed.

\section{siRNA, plasmid and transfection}

All transfection was performed using the TransIT-Xs Dynamic delivery system (Mirus, Madison, WI, USA) following the manufacturer's protocol. Transient knockdown of Ahnak and Bmpr1 $\alpha$ was performed in primary SVF and C3H10T1/2 cells. Pre-designed Ahnak, Bmpr1 $\alpha$ and control scrambled siRNA were purchased from BIONEER (Daejeon, Korea). Cells were transfected with $50 \mu \mathrm{M}$ siRNA. Full-length mouse complementary DNA sequence for Bmpr1 $\alpha$ was obtained from GeneCopoeia and subsequently cloned into the pEGFP-N1 (Stratagene, Santa Clara, CA, USA).

\section{Ahnak knock-out cell line}

Stable knock-out of LGALS3BP was performed in C3H10T1/2 cells. Cells were seeded in $60 \mathrm{~mm}$ cell culture dishes at a density of $5 \times 10^{5}$ cells per well. The next day, cells were transfected with Ahnak or control CRISPR/ Cas9 plasmid using the TransIT-Xs Dynamic delivery system. Transfected cells were collected by Cell Sorter (Sony, Tokyo, Japan) and cloned in 96 well culture plates for 2-3 weeks for selection of Ahnak Knock-out clones.

\section{Semi-quantitative RT-PCR}

RT-PCR analysis was performed, as described previously $^{36,37}$. Briefly, the total RNA was harvested from treated cells using TRIzol reagent (Invitrogen, Carlsbad, CA) following the manufacturer's protocol. One microgram of RNA was used as a template for reversetranscription using the Prime Script 1'st strand cDNA Synthesis kit (Takara, Kyoto, Japan). PCR was performed with 20 ng of cDNA using a PCR pre-mixture (Bioneer, Deajeon, Korea). The primers used are provided in Supplementary Table 1.

\section{Western blotting}

Western blotting analysis was performed, as described previously $^{36}$. Protein extraction of the cells were lysed in modified RIPA buffer (1 mM Tris-HCl, pH 7.4, $100 \mathrm{mM}$ $\mathrm{NaCl}, 1 \mathrm{mM}$ EDTA, $1 \mathrm{mM} \mathrm{NaF}, 2 \mathrm{mM} \mathrm{Na} 3 \mathrm{VO} 4$, protease inhibitor, $0.5 \%$ Deoxycholate, $1 \%$ NP-40 and $1 \%$ Triton $\mathrm{X}-100)$. Equal amounts of protein $(20-50 \mu \mathrm{g})$ were loaded in $8 \%$ or $10 \%$ sodium dodecyl sulfate-polyacrylamide gel electrophoresis (SDS-PAGE) and transferred by blotting to polyvinylidene fluoride membranes (PVDF; EMD
Millipore, Billerica, MA, USA). Detection was performed using EZ-Western Lumi Pico (DoGen, Seoul, Korea) according to the manufacturer's instructions.

\section{Immunohistochemistry and immunofluorescence}

The iWAT tissues were collected, fixed with formalin, and cut into 5- $\mu \mathrm{m}$ slices. Paraffin-embedded sections of iWAT were deparaffinized, rehydrated, and subjected to immunohistochemistry. For immunohistochemistry, sections were incubated with Ahnak and Bmpr1 $\alpha$ antibodies overnight at $4{ }^{\circ} \mathrm{C}$. Antibody detection was performed using the EnVision detection system (Dako North America Inc., Carpinteria, CA, USA) according to the manufacturer's protocol. For Immunofluorescence assays, cells were plated on 4-well chamber slides (Nunc, Rochester, NY, USA) and fixed in cold methanol at $-20^{\circ}$ $\mathrm{C}$ for $15 \mathrm{~min}$. Chamber slides were incubated overnight with specific antibodies at $4{ }^{\circ} \mathrm{C}$. Cells were washed three times in PBS containing 0.1\% Triton X-100 in PBS (PBS$\mathrm{T})$, then incubated with Alexa fluorochrome conjugated secondary for $2 \mathrm{~h}$. After PBS-T washing, slides were mounted using Fluoroshield with 4',6-diamidino-2-phenylindole (DAPI) mounting media (Immunobioscience, Mukiteo, WA, USA) and imaged by confocal microscopy (Zeiss, Oberkochen, Germany).

\section{Promoter assay}

Transfection was performed using TransIT-Xs dynamic delivery system (Mirus) as described above. Cells at $90 \%$ confluence were transfected with pEZX-PG04 for negative control, pEZX-Gapdh, pEZX-Bmpr1 $\alpha$, and pEZX-Bmpr2 promoter plasmid (Genecopoeia). After 2 days, supernatant were collected and kept at $-20{ }^{\circ} \mathrm{C}$ until assayed. Gaussia luciferase (GLuc) and secreted alkaline phosphatase (SEAP) were measured in supernatant using Secrete-Pair Dual Luminescence Assay Kit (Genecopoeia). SEAP activity was used for normalization such as an internal control for transfection efficiency.

\section{Statistical analysis}

Means and S.D. were calculated using Microsoft Excel software (Microsoft, Redmond, WA, USA). A two-tailed Student's $t$-test and unpaired Student's $t$-test were used for statistical analysis of comparative data. Values of $p<$ $0.05, p<0.01$, and $p<0.005$ were considered significant depending on the experiment. Pearson correlation $(p$ value $<0.05)$ and microarray data set (GSE28598) constructed by Yadav et al. was used to verify the coexpression patterns between Ahnak and TGF- signaling pathway gene sets.

\section{Acknowledgements}

We thank Dr. Y. H. Lee for the gift of the SV-40 LT/pSG5 encoded viral construction. This research was supported by Korea Mouse Phenotyping Project (2013M3A9D5072550) of the National Research Foundation (NRF) 
funded by the Ministry of Science and ICT. This research was also supported by a grant of the Korea Health Technology R\&D Project through the Korea Health Industry Development Institute (KHIDI), funded by the Ministry of Health \& Welfare, Republic of Korea (grant number: H113C2148) and partially supported by the Brain Korea 21 Plus Program and the Research Institute for Veterinary Science of Seoul National University.

\section{Author details}

${ }^{1}$ Korea Mouse Phenotyping Center (KMPC), Seoul National University, Seoul, Republic of Korea. ${ }^{2}$ Department of Surgery, University of Michigan, Ann Arbor, MI, USA. "'Laboratory of Developmental Biology and Genomics, College of Veterinary Medicine, Seoul National University, Seoul, Republic of Korea. ${ }^{4}$ National Cancer Center, Goyang-si, Gyeonggi-do, Republic of Korea. ${ }^{5}$ Interdisciplinary Program for Bioinformatics, Program for Cancer Biology and BIO-MAX institute, Seoul National University, Seoul 08826, Republic of Korea

\section{Conflict of interest}

The authors declare that they have no conflict of interest.

\section{Publisher's note}

Springer Nature remains neutral with regard to jurisdictional claims in published maps and institutional affliations.

Supplementary Information accompanies this paper at (https://doi.org/ 10.1038/s41419-018-0873-6).

Received: 23 January 2018 Revised: 8 June 2018 Accepted: 9 July 2018 Published online: 28 August 2018

\section{References}

1. Sun, K., Tordjman, J., Clement, K. \& Scherer, P. E. Fibrosis and adipose tissue dysfunction. Cell Metab. 18, 470-477 (2013).

2. Pfeifer, A. \& Hoffmann, L. S. Brown, beige, and white: the new color code of fat and its pharmacological implications. Annu. Rev. Pharmacol. Toxicol. 55, 207-227 (2015)

3. Scherer, P. E. Adipose tissue: from lipid storage compartment to endocrine organ. Diabetes 55, 1537-1545 (2006).

4. Rosen, E. D. \& Spiegelman, B. M. What we talk about when we talk about fat. Cell 156, 20-44 (2014).

5. Savage, D. B., Petersen, K. F. \& Shulman, G. I. Disordered lipid metabolism and the pathogenesis of insulin resistance. Physiol. Rev. 87, 507-520 (2007).

6. Peng, $X$. et al. Thioredoxin reductase 1 suppresses adipocyte differentiation and insulin responsiveness. Sci. Rep. 6, 28080 (2016).

7. Rosen, E. D. \& MacDougald, O. A. Adipocyte differentiation from the inside out. Nat. Rev. Mol. Cell Biol. 7, 885-896 (2006).

8. Young, $H$. E. et al. Mesenchymal stem cells reside within the connective tissues of many organs. Dev. Dyn. 202, 137-144 (1995).

9. Tang, Q. Q. \& Lane, M. D. Adipogenesis: from stem cell to adipocyte. Annu. Rev. Biochem. 81, 715-736 (2012).

10. Rosen, E. D. \& Spiegelman, B. M. Molecular regulation of adipogenesis. Annu. Rev. Cell Dev. Biol. 16, 145-171 (2000).

11. Rosen, E. D., Walkey, C. J., Puigserver, P. \& Spiegelman, B. M. Transcriptional regulation of adipogenesis. Genes Dev. 14, 1293-1307 (2000).

12. Tang, Q. Q., Otto, T. C. \& Lane, M. D. CCAAT/enhancer-binding protein beta is required for mitotic clonal expansion during adipogenesis. Proc. Natl Acad. Sci. USA 100, 850-855 (2003).

13. Urist, M. R. Bone: formation by autoinduction. Science 150, 893-899 (1965).

14. Bellusci, S., Henderson, R., Winnier, G., Oikawa, T. \& Hogan, B. L. Evidence from normal expression and targeted misexpression that bone morphogenetic protein (Bmp-4) plays a role in mouse embryonic lung morphogenesis. Development 122, 1693-1702 (1996).

15. Jones, C. M., Dale, L., Hogan, B. L., Wright, C. V. \& Smith, J. C. Bone morphogenetic protein-4 (BMP-4) acts during gastrula stages to cause ventralization of Xenopus embryos. Development 122, 1545-1554 (1996).

16. Miyazono, K., Kamiya, Y. \& Morikawa, M. Bone morphogenetic protein receptors and signal transduction. J. Biochem. 147, 35-51 (2010).

17. Mueller, T. D. \& Nickel, J. Promiscuity and specificity in BMP receptor activation. FEBS Lett. 586, 1846-1859 (2012).

18. Heldin, C. H., Miyazono, K. \& ten Dijke, P. TGF-beta signalling from cell membrane to nucleus through SMAD proteins. Nature 390, 465-471 (1997).

19. Massague, J. \& Wotton, D. Transcriptional control by the TGF-beta/Smad signaling system. EMBO J. 19, 1745-1754 (2000).

20. Huang, $H$. et al. BMP signaling pathway is required for commitment of $\mathrm{C} 3 \mathrm{H} 10 \mathrm{~T} 1 / 2$ pluripotent stem cells to the adipocyte lineage. Proc. Natl Acad. Sci. USA 106, 12670-12675 (2009).

21. Bowers, R. R., Kim, J. W., Otto, T. C. \& Lane, M. D. Stable stem cell commitment to the adipocyte lineage by inhibition of DNA methylation: role of the BMP-4 gene. Proc. Natl Acad. Sci. USA 103, 13022-13027 (2006).

22. Otto, T. C., Bowers, R. R. \& Lane, M. D. BMP-4 treatment of C3H10T1/2 stem cells blocks expression of MMP-3 and MMP-13. Biochem. Biophys. Res. Commun. 353, 1097-1104 (2007).

23. Shin, J. H. et al. Obesity resistance and enhanced insulin sensitivity in Ahnak-/mice fed a high fat diet are related to impaired adipogenesis and increased energy expenditure. PLOS ONE 10, e0139720 (2015).

24. Sekiya, F., Bae, Y. S., Jhon, D. Y., Hwang, S. C. \& Rhee, S. G. AHNAK, a protein that binds and activates phospholipase C-gamma1 in the presence of arachidonic acid. J. Biol. Chem. 274, 13900-13907 (1999).

25. Lee, I. H. et al. AHNAK-mediated activation of phospholipase C-gamma1 through protein kinase C. J. Biol. Chem. 279, 26645-26653 (2004).

26. Lee, I. H. et al. Ahnak protein activates protein kinase C (PKC) through dissociation of the PKC-protein phosphatase 2A complex. J. Biol. Chem. 283 6312-6320 (2008)

27. de Morree, A. et al. Self-regulated alternative splicing at the AHNAK locus. FASEB J. 26, 93-103 (2012).

28. Ramdas, M., Harel, C., Armoni, M. \& Karnieli, E. AHNAK KO mice are protected from diet-induced obesity but are glucose intolerant. Horm. Metab. Res. 47, 265-272 (2015)

29. Li, J., Yu, X., Pan, W. \& Unger, R. H. Gene expression profile of rat adipose tissue at the onset of high-fat-diet obesity. Am. J. Physiol. Endocrinol. Metab. 282, E1334-E1341 (2002)

30. Shin, J. H. et al. AHNAK deficiency promotes browning and lipolysis in mice via increased responsiveness to beta-adrenergic signalling. Sci. Rep. 6, 23426 (2016).

31. Yadav, H. et al. Protection from obesity and diabetes by blockade of TGF-beta/ Smad3 signaling. Cell Metab. 14, 67-79 (2011).

32. Jinek, M. et al. A programmable dual-RNA-guided DNA endonuclease in adaptive bacterial immunity. Science 337, 816-821 (2012).

33. Astori, G. et al. "In vitro" and multicolor phenotypic characterization of cell subpopulations identified in fresh human adipose tissue stromal vascular fraction and in the derived mesenchymal stem cells. J. Transl. Med. 5, 55 (2007).

34. Gao, Y. et al. Early adipogenesis is regulated through USP7-mediated deubiquitination of the histone acetyltransferase TIP60. Nat. Commun. 4, 2656 (2013).

35. Lee, M. R. et al. Lipolytic effect of novel extracts from mulberry (Morus alba) leaves fermented with Cordyceps militaris in the primary adipocytes derived from SD rats. Lab. Anim. Res. 33, 270-279 (2017)

36. Woo, J. K. et al. Mucin 1 enhances the tumor angiogenic response by activation of the AKT signaling pathway. Oncogene 31, 2187-2198 (2012).

37. Fungfuang, W., Terada, M., Komatsu, N., Moon, C. \& Saito, T. R. Effects of estrogen on food intake, serum leptin levels and leptin mRNA expression in adipose tissue of female rats. Lab. Anim. Res. 29, 168-173 (2013). 